culture counting and ATPlite assays we found the WT+ and NP lines to have a significant $(\mathrm{P}<0.01)$ decrease in growth compared to control and $\mathrm{PP}$ mutant. This was further supported by knockdown of MARCKS in a high expressing patient derived xenograft (PDX) line XD456 which resulted in increased neurosphere formation in a limiting dilution assay and increased cell growth by cell titer glow. We also found radiation sensitivity to enhance in the WT and NP expressing lines through a combination of clonogenic, comet and gamma-H2AX foci formation assays. Through orthotopic implantation of our MARCKS ED mutants into athymic nude mice, we saw a significant $(\mathrm{P}<0.05)$ survival benefit and increased response to radiation in our WT+ line with 6 Gy total dose delivered (2 Gy, 3X in a week). Overall, MARCKS phosphorylation alters its tumor suppressive effects.

\section{CSIG-11. ONCOSTREAMS: NOVEL STRUCTURES THAT SPECIFY GLIOMAS' SELF-ORGANIZATION, ARE ANATOMICALLY DISCRETE, FUNCTIONALLY UNIQUE, AND MOLECULARLY DISTINCT \\ Andrea Comba ${ }^{1}$, Dan Zamler ${ }^{1}$, Anna E Argento ${ }^{1}$, Carl Koschmann ${ }^{1}$,} Felipe J Nunez ${ }^{1}$, Marta Edwards ${ }^{1}$, Padma Kadiyala ${ }^{1}$, Neha Kamran ${ }^{1}$, Viveka Nand Yadav ${ }^{1}$, Sebastien Motsch ${ }^{2}$, Maria G Castro ${ }^{1,3}$ and Pedro R. Lowenstein ${ }^{1,3} ;{ }^{1}$ Dept. of Neurosurgery, University of Michigan Medical School, Ann Arbor, MI, USA, ${ }^{2}$ Department of Mathematics, Arizona State University, Tempe, AZ, USA, ${ }^{3}$ Dept. of Cell and Developmental Biology, University of Michigan Medical School, Ann Arbor, MI, USA

Whether tumors in general and gliomas in particular are self-organized has not been determined. Brain tumor self-organization would be revealed through individual recognizable structures. Here we demonstrate that brain tumors are indeed self-organized. Brain tumor self-organization is evidenced by the existence of oncostreams, multicellular structures composed of elongated glioma cells. Oncostream structure, function and molecular makeup was studied in several glioma models. In experimenta mouse models, and in human GBM tumors, oncostreams were demarcated anatomically as multicellular arrangements of fusiform cells, 10-20 cells wide, of various lengths and distributed throughout the tumors. Using genetically engineered glioma models we found a negative correlation between oncostream density and survival, suggesting that oncostreams contribute to tumor aggressiveness. As oncostreams also reached tumor borders, it is likely that they participate in glioma invasion. Further by placing slow moving glioma stem cells into oncostreams we detected that these function as streams that distribute glioma cells throughout the tumors. Importantly, we performed the molecular analysis of oncostreams using a Laser Microdissection Microscope to dissect oncostreams from surrounding tumor. The transcriptome landscape was analyzed by next generation sequencing using RNA-Seq and advanced bioinformatics algorithms. The molecular analysis showed that oncostreams are defined by a distinctive transcriptome landscape. We hereby show that oncostreams: (i) are anatomically discrete structures that reveal the existence of glioma self-organization, (ii) are functionally unique as they regulate glioma growth, invasion and spread, and (iii) molecularly distinct, their genetic landscape and associated gene networks differentiate oncostreams from surrounding glioma. Understanding glioma self-organization and its molecular basis will contribute to uncover novel targets for future translational development.

CSIG-12. EXPLORING THE FUNCTIONAL RELATIONSHIP BETWEEN CAPICUA (CIC) AND ATAXIN-1-LIKE (ATXN1L) IN GLIOMA

Derek Wong $^{1}$, Kohl Lounsbury ${ }^{1}$, Veronique LeBlanc ${ }^{1,2}$, Suganthi Chittaranjan ${ }^{1,2}$, Marco Marra ${ }^{2,3}$ and Stephen Yip ${ }^{4} ;{ }^{1}$ University of British Columbia, Vancouver, BC, Canada, ${ }^{2}$ BC Genome Science Center, Vancouver, BC, Canada, ${ }^{3}$ BC Cancer Agency, Vancouver, BC, Canada, ${ }^{4}$ Vancouver General Hospital, Vancouver, BC, Canada

BACKGROUND: Oligodendroglioma (ODG), a molecularly defined subtype of glioma, is a treatment responsive, slow growing tumour strongly associated with IDH mutation and $1 \mathrm{p} 19 \mathrm{q}$ co-deletion. Mutations in CIC, located on chromosome $19 \mathrm{q}$, have been found in up to $70 \%$ of IDH mutated, $1 \mathrm{p} 19 \mathrm{q}$ co-deleted ODGs; suggesting that loss or altered function of CIC may be crucially associated with ODG's unique biology. CIC and ATXN1L have previously been implicated in neurodegeneration, however, this interaction has not been studied in cancer. METHODS: CIC and ATXN1L interaction was confirmed using immunoprecipitation and immunofluorescence in multiple cell lines. CIC and ATXN1L knockout $(\mathrm{KO})$ cell lines were generated using CRISPR/Cas9 followed by gene expression profiling using Affymetrix microarray. Differential gene expression analysis was also performed on patient lower grade glioma (LGG) RNAseq data publicly available through TCGA. Functional biology experiments to further characterize CIC-ATXN1L interaction were also performed using KO cell systems. RESULTS: Immunoprecipita- tion and immunofluorescence confirmed CIC and ATXN1L interaction within the cell nucleus of multiple cell lines. Differential expression (DE) analysis of CIC KO, ATXN1L KO and TCGA LGG cohort converged on activation of the MAPK pathway and dysregulation of central nervous system development; with significant overlap in differentially expressed genes $(\mathrm{n}=110)$. Furthermore, experiments using our KO cell systems suggest a functionally reciprocal relationship between CIC and ATXN1L and regulation of their respective pathways. CONCLUSIONS: CIC and ATXN1L form a functionally reciprocal relationship where loss of one partner leads to dysregulation of both partners. Although CIC mutations are predominant in ODGs, the CIC-ATXN1L axis may be exploited in other gliomas and cancers which rely on maintaining MAPK signaling such as EGFR amplified glioblastoma and may be a target for future prognostics and therapeutics.

\section{CSIG-14. HEPARAN SULFATE MODULATES CELL SIGNALING BY RECEPTOR TYROSINE KINASES IN HUMAN AND MURINE GLIOBLASTOMA}

Yuki Ohkawa, Anna Wade, Olle R. Lindberg, Katharine Chen, Vy M. Tran and Joanna J. Phillips; Department of Neurological Surgery, Helen Diller Cancer Research Center, University of California, San Francisco, San Francisco, CA, USA

Heparan sulfate (HS) / Heparan sulfate proteoglycans (HSPGs) are implicated in tumor malignancy based on their ability to promote cell signaling and tumor invasion. The mechanisms by which they do this in an individual tumor type, however, are often poorly defined. Using both patient-derived glioblastoma (GBM) tumorsphere lines and a murine model for GBM, we investigate how cell surface HSPGs modulate cell signaling in infiltrating astrocytoma. In human tumorsphere lines, chemical ablation of cell surface HS reduced the phosphorylation of several receptor-tyrosine kinases (RTKs) including platelet-derived growth factor receptor $\alpha$ (PDGFR $\alpha$ ). The reduction in PDGFRA activation was associated with decreased binding of tagged PDGF-BB ligand to the cell surface suggesting HS can promote ligand retention and activity at the cell surface. To ablate all HS production we established HS-deficient murine tumor-prone cells by knockout of Ext1, a glycosyltransferase required for HS chain elongation. HS-deficient cells had decreased growth in vitro, decreased invasion in a 3D spheroid invasion assays, and reduced activation of RTKs as detected by Western blotting compared to control cells. Preliminary in vivo experiments suggest HS-deficient cells also exhibit reduced growth in vivo and the structural role for HS in RTK signaling in glioma can now be addressed.

CSIG-15. PROTEOMIC AND PHOSPHOPROTEOMIC ANALYSIS OF HUMAN MEDULLOBLASTOMA REVEALS DISTINCT ACTIVATED PATHWAYS BETWEEN SUBGROUPS

Antoine Forget ${ }^{1}$, Martignetti Loredana ${ }^{2}$, Sebastian Brabetz ${ }^{3}$, Daniel Picard ${ }^{4}$, Stephanie Puget ${ }^{5}$, Laurence Calzone ${ }^{2}$, Patrick Poullet ${ }^{2}$, Arnau Montagud ${ }^{2}$, Stephane Liva ${ }^{2}$, Florent Dingli ${ }^{6}$, Guillaume Arras ${ }^{6}$, Hua $\mathrm{Yu}^{1}$, Audrey Mercier ${ }^{1}$, Celio Pouponnot ${ }^{7}$, Damarys Loew ${ }^{6}$, Franck Bourdeaut ${ }^{8}$, Christelle Dufour ${ }^{9}$, Pascale Varlet ${ }^{10}$, Michael Taylor $^{11}$, Marcel Kool ${ }^{12}$, Stefan M. Pfister ${ }^{13}$, Emmanuel Barillot ${ }^{2}$, Marc Remke ${ }^{14}$ and Ayrault Olivier ${ }^{1} ;{ }^{1}$ "Signaling in development and brain tumors" Institut Curie Curie / CNRS UMR 3347 / INSERM U1021, Orsay, France, ${ }^{2}$ Computational and Systems Biology of Cancer, Institut Curie, Paris, France, Paris, France, ${ }^{3}$ Division of Pediatric Neurooncology, German Cancer Research Center (DKFZ), Heidelberg, Germany, ${ }^{4}$ Division of Neurosurgery, Arthur and Sonia Labatt Brain Tumour Research Center, University of Toronto, Toronto, ON, Canada, ${ }^{5}$ AP-HP, Department of Neurosurgery, Necker-Enfants Malades Hospital, Université René Descartes, Paris, France, ${ }^{6}$ Proteomics and Mass Spectrometry Laboratory, Institut Curie, PSL Research University, Paris, France, ${ }^{7}$ "Raf and Maf Signaling in Oncogenesis and Development" Institut Curie Curie / CNRS UMR 3347 / INSERM U1021, France, Paris, France, ${ }^{8}$ Paris SciencesLettres Research University, Institut Curie, Departement d'Oncologie Pediatrique-Adolescents et Jeunes adultes, Paris, France, ${ }^{9}$ Gustave Roussy, Paris, France, ${ }^{10} \mathrm{Hoppital}$ Sainte-Anne, Paris, France, ${ }^{11}$ Division of Neurosurgery,Hospital for Sick Children, Toronto, ON, Canada, ${ }^{12}$ German Cancer Research Center, Heidelberg, Heidelberg, Germany, ${ }^{13}$ Division of Pediatric Neurooncology, Pre-Clinical program, Hopp Children's Cancer Center at the NCT (KiTZ), German Cancer Research Center (DKFZ), and German Cancer Consortium (DKTK), Heidelberg, Germany, ${ }^{14}$ Deutsches Krebsforschungszentrum (DKFZ), Dusseldorf, Germany

Deregulations in fundamental signaling pathways are key events in pathogenesis of cancer. One intriguing illustration that still holds blind spots is the pediatric brain tumor arising from the developing cerebellum: medulloblastoma (MB). Extensive high-throughput sequencing led to the characterization of four MB subgroups (WNT, SHH, Group 3 and Group 4) delineated with distinct molecular signatures and clinical outcomes. However, up-to- 